\title{
Wide Excision Without Radiation for Desmoplastic Melanoma
}

\author{
Alisha Arora, M.D. ${ }^{1}$ \\ Lori Lowe, M.D. ${ }^{2,3}$ \\ Lyndon Su, M.D. ${ }^{2,3}$ \\ Riley Rees, M.D. ${ }^{1}$ \\ Carol Bradford, M.D. ${ }^{4}$ \\ Vincent C. Cimmino, M.D. ${ }^{1}$ \\ Alfred E. Chang, M.D. ${ }^{1}$ \\ Timothy M. Johnson, M.D. ${ }^{1,3,4}$ \\ Michael S. Sabel, M.D. ${ }^{1}$ \\ ${ }^{1}$ Department of Surgery, University of Michigan, \\ Ann Arbor, Michigan.

\footnotetext{
${ }^{2}$ Department of Pathology, University of Michigan, Ann Arbor, Michigan.
}
${ }^{3}$ Department of Dermatology, University of Mich- igan, Ann Arbor, Michigan.

${ }^{4}$ Department of Otolaryngology, University of Michigan, Ann Arbor, Michigan.
BACKGROUND. Adjuvant radiation has been proposed for the treatment of patients with desmoplastic melanoma, who reportedly have local recurrence rates as high as $40-60 \%$. The authors investigated local recurrence rates at a tertiary referral center to determine the success of wide excision alone for patients with desmoplastic melanoma.

METHODS. A review of a prospectively maintained melanoma clinical data base identified 65 patients between March 1997 and March 2004 with pure cutaneous desmoplastic melanoma. Complete surgical, histopathologic, and staging information was collected along with data on outcome, including local, regional, and distant recurrence and survival.

RESULTS. Similar to previous reports, patients with desmoplastic melanoma had a male-to-female ratio of 2 to 1, a mean age of 65.0 years (range, 31-92 yrs), and the majority of their tumors (55\%) were located on the head and neck. The mean Breslow depth at diagnosis was $4.21 \mathrm{~mm}$, with $38 \%$ of tumors thicker than $4.0 \mathrm{~mm}$. All patients in this series underwent wide excision without radiation therapy. Surgical margins $\leq 2 \mathrm{~cm}$ were obtained for all trunk and extremity lesions and for $63 \%$ of head and neck lesions that measured $>1 \mathrm{~mm}$ in depth (63\%). Margins of 1-2 cm were obtained for the remaining patients. Among 49 patients who had a minimum of 2 years of follow-up (mean, $3.7 \mathrm{yrs}$ ), the local recurrence rate was $4 \%$ ( 2 of 49 patients). Seventy-eight percent of the patients studied remained alive with no evidence of disease.

CONCLUSIONS. Local recurrence rates in the current series were considerably lower than the historically reported rates. This finding suggests that, for patients with desmoplastic melanoma, wide local excision with careful attention to appropriate margins produces excellent local control rates without the need for adjuvant radiation. Cancer 2005;104:1462-7. (C) 2005 American Cancer Society.

KEYWORDS: melanoma, desmoplastic, neurotropic, radiation, local recurrence.

- ince it was described originally by Conley et al. in 1971, desmo- plastic melanoma has been reported as a locally aggressive tumor in the literature. ${ }^{1}$ Conley and colleagues described this melanoma subtype as typically a bulky, subcutaneous tumor that recurred frequently and metastasized often. In view of the dense fibrosis accompanying the spindle cell tumor, those authors proposed the term desmoplastic malignant melanoma. Eight years later, Reed and Leonard noted that spindle cell melanomas exhibiting desmoplasia also demonstrated neurotropism and a "dominant neuroid appearance"2 and proposed the term neurotropic melanoma for this variant of spindle cell melanoma. Today, it is recognized that both desmoplastic melanoma and neurotropic melanoma represent rare variants of melanoma that are part of a histologic continuum.

Desmoplastic melanoma is regarded as a locally aggressive mel- 
anoma subtype with local recurrence rates as high as $40-60 \%{ }^{3-6}$ For this reason, adjuvant radiotherapy has been advocated by some for local control. ${ }^{6,7}$ A Phase II trial to evaluate the benefit of adjuvant radiation has been initiated by the North Central Cancer Treatment Group. Because our institution does not utilize adjuvant radiation routinely in the management of melanoma, we sought to determine local recurrence rates for patients with desmoplastic melanoma who underwent wide excision alone. Here, we report that, with wide excision alone and careful pathologic examination of margins, excellent local control of desmoplastic melanoma can be achieved.

\section{MATERIALS AND METHODS}

A review was performed of a prospectively maintained melanoma clinical data base at the University of Michigan Multidisciplinary Melanoma Clinic with Institutional Review Board approval. Between March 1997 and March 2004, 79 patients were identified with pure desmoplastic/desmoplastic neurotropic melanoma or other histologic subtypes with desmoplastic features. Clinical data were reviewed for all patients. Histopathologic review of all tumor specimens, including materials from referring institutions, was conducted by dermatopathologists at the University of Michigan. Of the 79 lesions identified, 66 were pure desmoplastic or desmoplastic/neurotropic melanomas. A pure desmoplastic melanoma was defined as melanoma composed of variably atypical, spindled-to-fusiform melanocytes associated with a prominent collagenous or desmoplastic stroma, with or without neurotropism. Spindle cell melanomas without intratumoral desmoplasia were excluded from this study. Sixty-five lesions were cutaneous, and 1 lesion was mucosal (retromolar). The current review was limited to the 65 cutaneous pure desmoplastic melanomas, with and without neurotropism.

All patients underwent definitive management at the University of Michigan with wide local excision. For all melanomas that measured $>2.0 \mathrm{~mm}$ in Breslow depth, the objective of wide excision was a margin of $2.0 \mathrm{~cm}$. For lesions between $1.0 \mathrm{~mm}$ and 2.0 $\mathrm{mm}$ in Breslow depth, margins of at least $1.0 \mathrm{~cm}$ were obtained if $2.0-\mathrm{cm}$ margins were not possible. Margins of $1.0 \mathrm{~cm}$ were taken around lesions that measured $<1.0 \mathrm{~mm}$ in depth. Forty-three patients $(67 \%)$ underwent lymphatic mapping with sentinel lymph node biopsy (SLNB). The technique of SLNB has been described previously. ${ }^{8}$ Follow-up information from the date of definitive treatment was obtained through the University of Michigan Cancer Registry.
TABLE 1

Clinical and Pathologic Characteristics of 65 Patients with Desmoplastic Melanoma

\begin{tabular}{lc}
\hline Characteristic & No. of patients (\%) \\
\hline Gender & \\
Male & $44(68)$ \\
Female & $21(32)$ \\
Age & \\
$<40$ yrs & $5(8)$ \\
$40-50$ yrs & $11(17)$ \\
$50-60$ yrs & $11(18)$ \\
$60-70$ yrs & $12(18)$ \\
$>70$ yrs & $26(40)$ \\
Location & $36(55)$ \\
Head and neck & $12(18)$ \\
Trunk & $13(20)$ \\
Upper extremity & $4(6)$ \\
Lower extremity & \\
Breslow depth & $7(11)$ \\
$<1.00$ mm & $19(29)$ \\
$1.01-2.00$ mm & $14(21)$ \\
2.01-4.00 mm & $25(38)$ \\
$>4.01$ mm & \\
Neurotropism & $44(68)$ \\
Absent & $21(32)$ \\
Present & \\
Ulceration & $53(82)$ \\
Absent & $9(14)$ \\
Present & $3(5)$ \\
Unknown & \\
Mitotic rate & $28(43)$ \\
$<1 / \mathrm{mm}^{2}$ & $23(35)$ \\
$1-5 / \mathrm{mm}^{2}$ & \\
$>5 / \mathrm{mm}^{2}$ & \\
Unknown & \\
& \\
\hline & \\
\hline
\end{tabular}

\section{RESULTS}

We identified 65 patients with pure desmoplastic melanoma who were treated at the University of Michigan between 1997 and 2004 (Table 1). Neurotropism was present in 21 lesions (32\%) and was absent in 44 lesions (68\%). Forty-four patients were male, and the male-to-female ratio was 2.1:1.0. The median age at presentation was 65.0 years (range, 31-92 yrs). Seventyfive percent of the patients were age 50 years or older. More than one-half of the lesions (55\%) were located on the head and neck. Eighteen percent of the lesions were located on the trunk, $20 \%$ were located on the upper extremity, and only 4 lesions (6\%) were located on the lower extremity.

It was rare for desmoplastic melanoma to be detected at a thin stage. Only 7 patients (11\%) had melanomas that measured $<1.0 \mathrm{~mm}$. Twenty-five patients $(38 \%)$ had thick melanomas $(>4.0 \mathrm{~mm})$. Six of the seven thin melanomas were located on easily visible areas (two on the neck, one on the forehead, and three on the arm). In contrast, the thicker melanomas 
were located more often on the scalp, posterior neck, or back (13 of 25 thick melanomas were in these locations). Despite the advanced Breslow depth, other histologic prognostic factors were not dramatically worse. Ulceration was present in only 9 lesions (14\%), and 28 lesions ( $42 \%$ ) had a mitotic rate $<1 / \mathrm{mm}^{2}$. Even among the 25 thick melanomas, 18 lesions had no ulceration, and 15 lesions had mitotic rates $<1 / \mathrm{mm}^{2}$.

For the current review, we included pure desmoplastic melanoma with or without neurotropism. Neurotropism was present in 21 lesions (32\%). Age and gender distribution was equal between desmoplastic melanomas with or without neurotropism. Neurotropism was found less commonly when the melanoma was located on the extremities versus lesions located on the trunk or head and neck ( $15 \%$ vs. $32 \%$ ). Desmoplastic neurotropic melanomas had a thicker Breslow depth at presentation than desmoplastic melanoma without neurotropism $(5.96 \mathrm{~mm}$ vs. $3.05 \mathrm{~mm} ; P$ $=0.0006)$. There was no difference in the presence of ulceration or the mitotic rate.

All patients underwent a wide excision. For lesions on the head and neck, a margin $\geq 2.0 \mathrm{~cm}$ was obtained in 20 of 32 patients who had lesions that measured $>1.0 \mathrm{~mm}$ in depth $(63 \%)$. Of the remaining 12 patients, margins $<2.0 \mathrm{~cm}$ but $>1.0 \mathrm{c} \mathrm{m}$ were obtained, and 8 of those patients had lesions that measured between $1.00 \mathrm{~mm}$ and $2.00 \mathrm{~mm}$ in Breslow depth. In one patient, only narrow margins were taken near the eyelid. The three patients who had head and neck lesions that measured $<1.0 \mathrm{~mm}$ had $1.0-\mathrm{cm}$ excision margins; and, in 1 patient, the margins of resection were unknown. To obtain these margins, almost all patients ( 30 of 36 patients) who had head and neck lesions required reconstruction with a complex closure, advancement flaps, or skin grafts. For lesions on the trunk or extremities, $2.0-\mathrm{cm}$ margins were obtained for all patients who had melanomas that measured $>2.0 \mathrm{~mm}$ in Breslow depth. Five patients required split-thickness skin grafts for closure. One patient who had a desmoplastic melanoma on the arm that measured $0.6 \mathrm{~mm}$ in Breslow depth had margins of $1.0 \mathrm{~cm}$; however, the patient underwent reexcision, because the trailing edge of melanoma in situ was present in the peripheral margins.

One of 65 patients who presented with clinically involved lymph nodes underwent a complete lymph node dissection at the time of wide local excision. This patient had a 19-mm lesion located on the upper back with a palpable lymph node in the right axilla. Two of 26 lymph nodes were positive on axillary lymph node dissection. The patient had an in-transit recurrence 8 months later but, after resection, remained alive without evidence of recurrence 1 year later. Of the remain- ing 64 patients, 43 patients $(67 \%)$ underwent SLNB. Four patients had positive SLNB results (9\%). With an overall mean follow-up of 2.7 years, 2 patients who had negative SLNB results developed regional recurrences. One patient had a forehead lesion that measured $9 \mathrm{~mm}$ in Breslow depth and had a negative preauricular sentinel lymph node. He developed a recurrence with simultaneous cervical and pulmonary metastases and died of disease. The second patient, who had a lower back lesion that measured $7 \mathrm{~mm}$ in Breslow depth with a negative inguinal sentinel lymph node, developed recurrent disease in the iliac lymph nodes. He remained disease-free after undergoing superficial and deep inguinal dissection. This resulted in a false-negative rate of $33 \%$ ( 2 false negative results among 6 lymph node-positive patients) and a negative predictive value of $95 \%$. Of the 21 patients who underwent wide excision alone, 1 patient had a regional recurrence: He developed distant disease shortly after that and died of his disease.

Of the 44 patients who had lesions without neurotropism, 1 patient was clinically lymph node positive, and 28 patients underwent SLNB, which produced 1 positive result. To date, no patient has developed a regional recurrence. Of the 21 patients who had lesions with neurotropism, 15 patients underwent SLNB, which produced 3 positive results. Both regional recurrences after a negative SLNB were in patients who had lesions with neurotropism, which was the same as the regional recurrence in the patient who underwent wide excision alone. Therefore, with a median follow-up of 2.7 years, lymph node metastases were present in only $4 \%$ of patients who had desmoplastic melanomas without neurotropism but were present in $28 \%$ of patients who had desmoplastic melanomas with neurotropism $(P=0.01)$.

Among the cohort of 65 patients with pure desmoplastic melanoma who underwent wide local excision alone without radiation therapy, 5 patients were lost to follow-up, and 11 patients were treated within the past 2 years (none of whom has had a local recurrence). This leaves 49 patients with a minimum of 2 years of follow-up after therapy and a mean follow-up of 3.6 years. This subset has a profile similar to that of the full cohort (Table 2). Among these patients, there have been only 2 local recurrences (4\%), both of which were head and neck lesions. The first local recurrence was in a patient who had a lesion on the scalp that measured $8.5 \mathrm{~mm}$ in Breslow depth. The second local recurrence was in a patient who had a lesion on the temple that measured $5.0 \mathrm{~mm}$ in Breslow depth. Both recurred adjacent to the previous scar or skin graft site. Of the two local recurrences, one patient had neurotropism in the primary tumor and one did not. 
TABLE 2

Recurrence Rates after Definitive Management of Desmoplastic Melanoma in 49 Patients with a Minimum of 2 Years of Follow-Up

\begin{tabular}{lc}
\hline Characteristic & No. of patients (\%) \\
\hline Location & \\
Head and neck & $26(53)$ \\
Trunk & $9(18)$ \\
Upper extremity & $10(20)$ \\
Lower extremity & $4(8)$ \\
Male:female ratio & $2.5: 1.0$ \\
Age in yrs & \\
Median & 64.3 \\
Range & $21-92$ \\
Local recurrence rate & $2(4)$ \\
Distant recurrences & $3(6)$ \\
Died without disease & $6(11)$ \\
Two-yr disease-free survival (\%) & 78
\end{tabular}

Both patients underwent resection of their recurrences and remain disease free. Three patients developed distant metastases and died of their disease $(6 \%)$, and 6 patients died of other causes (12\%). Thirty eight patients $(78 \%)$ remain alive with no evidence of disease.

\section{DISCUSSION}

Desmoplastic melanoma is an uncommon variant of cutaneous melanoma, comprising approximately $4 \%$ of lesions. ${ }^{9}$ Several reports of desmoplastic melanoma have painted a uniform picture of the disease: specifically, these lesions are more common on the head and neck, they occur more often in men than in women, and they affect an older age group. The disease rarely is diagnosed early, often because it lacks the classic physical findings associated with melanoma and more frequently is amelanotic. In this regard, our series from the University of Michigan conforms to this description. Concordant with the prior observations, our population of patients with desmoplastic melanoma demonstrated a male preponderance of 2:1 and a considerably older age at presentation, with a median age of 65 years. Head and neck was the most common site disease by far, and they presented at an advanced Breslow depth, with a mean depth of $4.22 \mathrm{~mm}$.

In addition to the clinical characteristics described above, it has been shown that desmoplastic melanoma has an unusually high local recurrence rate and a low rate of metastasis to regional lymph nodes. Based on these observations, some even have suggested that desmoplastic melanoma has more in common with soft tissue sarcomas than cutaneous melanoma. ${ }^{10,11}$ Because of the reportedly high local recurrence rates, some authors have suggested surgi- cal margins of up to $3.0 \mathrm{~cm}^{4,5,9,12}$ with the use of adjuvant radiation. ${ }^{6,7}$

Indeed, desmoplastic melanoma does tend to have a lower incidence of regional metastases. Seven patients in the current series had regional disease $(4$ detected on SLNB, 1 clinically evident, and 3 regional recurrences), for an overall incidence of $11 \%$. Although regional disease is less frequent in desmoplastic melanoma, SLNB still is warranted in clinically lymph node-negative patients. Lymph node metastasis (as evidenced either by a positive sentinel lymph node or by clinical lymph node involvement) was significantly more common when neurotropism was present in the primary tumor. However, this may be related to the thicker Breslow depth seen in desmoplastic melanomas with neurotropism.

The primary focus of this review was the local recurrence rate after wide local excision without radiation, and our results did not confirm the rates described by previous studies. The mean time to local recurrence after wide excision is 12 months $^{6}$; and Quinn et al., in their review of 280 patients, found that $>75 \%$ of all recurrences occurred within 2 years. ${ }^{9}$ For this reason, we limited our evaluation to those patients who had a minimum of 2 years follow-up (49 of 65 patients). Of these 49 patients, only 2 patients had local recurrences (4\%). A similar finding was reported recently by investigators at Memorial Sloan-Kettering Cancer Center, who also found a local recurrence rate of $4 \%$ ( 1 of 24 patients). ${ }^{10}$ Similar to our procedure, they perform wide local excision of at least $2 \mathrm{~cm}$ when possible and do not use local adjuvant radiation therapy routinely. Like all retrospective research, it is possible to introduce biases. In the current study, it is possible that there may have been local recurrences in the 5 patients who were lost to follow-up. However, even in the unlikely situation that all 5 of those patients developed local recurrences, the local recurrence rate would be $13 \%$ ( 7 of 54 patients), which still is substantially lower than suggested in the literature.

Although there are several reports in the literature concerning desmoplastic melanoma that describe similar presentations (elderly patients, male preponderance, head and neck lesions), they vary widely in their description of local recurrence rates. The reasons for this are not entirely clear. One explanation may be the length of follow-up. However, because most local recurrences will occur within 2 years, ${ }^{9}$ it is unlikely that this is the only explanation. Another explanation may be the definition of desmoplastic melanoma. Establishing a correct histologic diagnosis is paramount and not always straightforward. Dermatopathologists with experience in the diagnosis of melanoma are needed, because desmoplastic melanoma can be con- 
TABLE 3

Local Recurrence Rates after Wide Excision of Desmoplastic Melanoma

\begin{tabular}{|c|c|c|c|c|c|}
\hline Institution (reference) & Yr & $\begin{array}{l}\text { Follow-up } \\
\text { (mos) }\end{array}$ & $\begin{array}{l}\text { No. of } \\
\text { patients }\end{array}$ & $\begin{array}{l}\text { LR rate } \\
(\%)\end{array}$ & Comments \\
\hline MSKCC (Gyorki et al., $2003^{10}$ ) & 2003 & 27 (median) & 27 & 4 & $\begin{array}{l}\text { The } 1 \text { LR was seen in a patient who underwent } \\
\text { excision of an 8-mm Clark Level V cheek } \\
\text { melanoma with a close but negative deep } \\
\text { margin at original excision }\end{array}$ \\
\hline University of Michigan & 2004 & 42 (median) & 49 & 4 & $\begin{array}{l}\text { Both LRs were thick melanomas on the head and } \\
\text { neck }\end{array}$ \\
\hline Sydney Melanoma Unit (Quinn et al., 19989) & 1998 & $\mathrm{NA}$ & 280 & 11 & $\begin{array}{l}\text { The LR rate was higher when the surgical } \\
\text { clearance margin was }<1 \mathrm{~cm} \text { and when } \\
\text { neurotropism was present }\end{array}$ \\
\hline University of South Florida (Payne et al., 2001 ${ }^{18}$ ) & 2001 & 18 (mean) & 30 & 23 & Margins of excision are not described \\
\hline Massachusetts General Hospital (Carlson et al., 1995 ${ }^{19}$ ) & 1995 & 24 (median) & 28 & 25 & $\begin{array}{l}\text { Two of } 7 \text { locally recurring lesions had only positive } \\
\text { margin biopsies, } 1 \text { had margins }<1 \mathrm{~cm} \text {, and } 1 \\
\text { had unknown margins }\end{array}$ \\
\hline University of Queensland (Smithers et al., 1992 ${ }^{12}$ ) & 1992 & 30 (median) & 58 & 29 & Margins of excision were unknown \\
\hline Mayo Clinic (Jaroszewski et al., $2001^{3}$ ) & 2001 & $\mathrm{NA}$ & 59 & 39 & $\begin{array}{l}\text { LR correlated with positive, unknown, or }<1 \mathrm{~cm} \\
\text { margins }\end{array}$ \\
\hline $\begin{array}{l}\text { University of California at San Francisco (Egbert et al., } \\
1988^{4} \text { ) }\end{array}$ & 1988 & 32.4 (mean) & 25 & 48 & $\begin{array}{l}\text { Eight of } 12 \text { patients with locally recurrent lesions } \\
\text { either were diagnosed initially with a benign } \\
\text { lesion or did not undergo histologic } \\
\text { examination of the initial excision specimen; } \\
\text { recurrence may be related to inadequate initial } \\
\text { therapy }\end{array}$ \\
\hline Queen Elizabeth Hospital (Jain and Allen, $1989^{5}$ ) & 1989 & 55 (mean) & 45 & 55 & $\begin{array}{l}\text { Margins of excision were unknown, and several } \\
\text { lesions were excised with positive or close } \\
\text { margins; recurrence may be related to } \\
\text { inadequate initial therapy }\end{array}$ \\
\hline
\end{tabular}

fused with scar, blue nevus, neurofibroma, dermatofibroma, schwannoma, fibromatosis, or spindle cell sarcoma. Failure to diagnose properly may lead to a high risk of local recurrence due to inadequate margins. It is possible that some older series with high local recurrence rates may have included some of these other lesions. In the current series, all pathologic specimens were reviewed to confirm the diagnosis of desmoplastic melanoma.

The most likely possibility for the discrepancy in high recurrence rates described may be attributable to the margins obtained at surgery and/or the histopathologic examination of those margins. Because many of these lesions will occur in the head and neck region, surgeons may be hesitant to obtain margins of $1-2 \mathrm{~cm}$. It has been well documented that local recurrence is significantly greater for desmoplastic melanoma when margins $<1 \mathrm{~cm}$ are obtained. ${ }^{9,12}$ In many series that reported high local recurrence rates without radiation, the surgical margins obtained were not quantifiable; were described only as "negative" or "unknown"; and, in several patients, had positive margins but were not reexcised (Table 3). Often, these series included patients who underwent "excision" at outside hospitals and who presented at the time of their local recurrence, which may result in a false elevation of the tertiary hospital's recurrence rate.

In addition to obtaining adequate margins in the operating room, careful histopathologic review of the specimen is necessary. Desmoplastic melanoma often arises within lentigo maligna. ${ }^{13}$ Not infrequently, the trailing edge of lentigo maligna subtly blends with surrounding, up-regulated melanocytes in sun-damaged skin, complicating the histologic assessment of the surgical margins. Failure to recognize and identify this atypical junctional melanocytic hyperplasia, which often is part of the trailing edge of lentigo maligna, may result in inadequate peripheral margin control and a theoretically higher rate of local recurrence. ${ }^{14,15}$ Neurotropism also is a histologic feature that has been associated with increased local recurrence rates. ${ }^{4,5,9,16}$ The neurotropic variant has a predilection for invasion along nerves. Thus, careful examination of the specimen for the presence of neurotropism and for perineural involvement at the resection margins is important to obtain adequate surgical margins and prevent local recurrence. In the current series, neurotropism was not associated with 
an increased local recurrence rate, although it did correlate with lymph node metastases.

Also, it is not always easy to identify the extent of desmoplastic melanoma on histopathologic examination. In some tumors, the cells demonstrate only mild atypia and can have a subtle, infiltrative pattern of growth. If margins $<2.0 \mathrm{~cm}$ are taken to avoid a skin graft or because of proximity to difficult structures, then a detailed histopathologic examination of all surgical margins should be performed. Reexcision is recommended for any areas in which the lesion approximates a margin, including for early lentigo maligna or atypical junctional melanocytic hyperplasia. In several series that demonstrated high local recurrence rates, many of these areas were attributed to the failure to recognize and obtain clear surgical margins. ${ }^{9,12,17}$

Based on previous reports, the use of adjuvant radiotherapy has been recommended after surgery to reduce local recurrence rates. A Phase II trial of postoperative radiotherapy after wide local excision is being initiated by the North Central Cancer Treatment Group. The low local recurrence rate achieved in the current study may provide useful information with respect to what local recurrence rate with adjuvant radiation therapy would justify the further evaluation of postoperative radiation in a Phase III trial. The results of these recent reviews suggest that excellent local control may be obtained with surgery alone. Although this may require the use of $2.0-\mathrm{cm}$ surgical margins for lesions that measure $>1.0 \mathrm{~mm}$ in Breslow depth and a more thorough histopathologic review of the surgical specimen, which is not without added cost and time, it certainly is worth considering, because it may improve local control rates and spare patients the cost and morbidity of adjuvant radiotherapy.

Local recurrences are more common for head and neck melanomas, possibly due to the inherent difficulties of wide local excision at this anatomic site. Because lesions on the trunk and extremities can be resected easily with $2-\mathrm{cm}$ margins, there should be little indication for radiotherapy after patients undergo resection with clear margins and with careful histologic assessment of trunk or extremity desmoplastic melanomas. In anatomically critical regions of the head and neck, the surgeon should attain up to 2-cm margins whenever feasible, and every effort should be made to clear the surgical margin. When anatomic considerations prevent the surgeon from achieving adequate surgical margins due to proximity to critical structures or after resection of a locally recurrent desmoplastic melanoma, radiation may be considered and potentially may be beneficial. Radiation also should be considered for lesions that are unresectable at presentation. Otherwise, the routine use of radiation as an adjuvant to surgery for desmoplastic melanoma, even for head and neck lesions, should not be necessary.

\section{REFERENCES}

1. Conley J, Lattes R, Orr W. Desmoplastic malignant melanoma (a rare variant of spindle cell melanoma). Cancer. 1971;28:914-936.

2. Reed JG, Leonard DD. Neurotropic melanoma: a variant of desmoplastic melanoma. Am J Surg Pathol. 1979;3:301-311.

3. Jaroszewski DE, Pockaj BE, DiCaudo DJ, Bite U. The clinical behavior of desmoplastic melanoma. Am J Surg. 2001;182: 590-595.

4. Egbert B, Kempson R, Sagebiel RW. Desmoplastic malignant melanoma. A clinicohistopathologic study of 25 cases. Cancer. 1988;62:2033-2041.

5. Jain S, Allen PW. Desmoplastic malignant melanoma and its variants. A study of 4 cases. Am J Surg Pathol. 1989;13:358373.

6. Vongtama R, Safa A, Gallardo D, et al. Efficacy of radiation therapy in the local control of desmoplastic melanoma. Head Neck 2003;25:423-428.

7. Anderson TD, Weber RS, Guerry D, et al. Desmoplastic neurotropic melanoma of the head and neck: the role of radiation therapy. Head Neck. 2002;24:1068-1071.

8. Su LD, Fullen DR, Lowe L, et al. Desmoplastic and neurotropic melanoma. Analysis of 33 patients with lymphatic mapping and sentinel node biopsy. Cancer. 2003;100:598604.

9. Quinn MJ, Crotty KA, Thompson JF, et al. Desmoplastic and desmoplastic neurotropic melanoma. Experience with 280 patients. Cancer. 1998;83:1128-1135.

10. Gyorki DE, Busam KJ, Panageas KS, et al. Sentinel lymph node biopsy for patients with cutaneous desmoplastic melanoma. Ann Surg Oncol. 2003;10:403-407.

11. Jaroswewski DE, Pockaj BE, DiGaudo DJ, et al. The clinical behavior of desmoplastic melanoma. Am J Surg. 2001;182: 590-595.

12. Smithers BM, McLeod GR, Little JH. Desmoplastic melanoma: patterns of recurrence. World J Surg. 1992;16:186190.

13. Clark WH, Elder DE, Van Horn M. The biologic forms of malignant melanoma. Hum Pathol. 1984;17:443-450.

14. Anderson KW, Baker SR, Lowe L, et al. Treatment of head and neck melanoma, lentigo maligna subtype: a practical surgical technique. Arch Facial Plast Surg. 2001;3:202-206.

15. Agarwal-Antal N, Bowen GM, Gerwels JW. Histologic evaluation of lentigo maligna with permanent sections: implications regarding current guidelines. J Am Acad Dermatol. 2002;47:743-748.

16. Baer SC, Schultz D, Synnestvedt M, et al. Desmoplasia and neurotropism: prognostic variables in patients with Stage I melanoma. Cancer. 1995;76:2242-2247.

17. Wharton JM, Carlson JA, Mihm MC. Desmoplastic malignant melanoma: diagnosis of early clinical lesions. Hum Pathol. 1999;30:537-542.

18. Payne WG, Kearney R, Wells K, et al. Desmoplastic melanoma. Am Surg. 2001;67:1004-1006.

19. Carlson JA, Dickersin GR, Sober AJ, Barnhill RL. Desmoplastic neurotropic melanoma. A clinicopathologic analysis of 28 cases. Cancer. 1995;75:478-494. 